\title{
ROBUST DESIGN OF THERMALLY ACTUATED MICRO- CANTILEVER USING NUMERICAL SIMULATIONS
}

\author{
Komeili, M. \& Menon, C. \\ MENRVA Research Group, School of Engineering Science, Simon Fraser University, Metro \\ Vancouver, Canada \\ E-Mail: mkomeili@sfu.ca, carlo_menon@sfu.ca
}

\begin{abstract}
Dynamic behaviour of a micro-cantilever beam under periodic electro-thermal loading is studied in this paper. For certain applications the beam is required to vibrate at a particular frequency. Modal analysis using 3D finite element is used in order to find the geometrical parameters that makes fundamental frequency of the beam match the required frequency. Then non-linear dynamic thermoelastic analysis is conducted on the system to analyse the time-history (transient) behaviour of the beam and record its tip displacement. However, due to uncertainties and non-repeatabilities that are inherent properties of the system along with those associated with the manufacturing, final product is likely to have deviations from these estimated values (fundamental frequency and tip displacement). Thus, choosing a nominal (desired) design and studying the deviation in natural frequency and tip displacement via $2^{k}$ factorial Design-of-Experiments (DOE), effect of uncertainties on the overall performance of the system is investigated. This allows finding the significance of individual parameters on the overall robustness of the design as well as potential interactions between various parameters. Finally, the expected behaviour of the micro-cantilever and its robustness to design and implementation uncertainties are elaborated and statements for robust design of this system are made. (Received in July 2015, accepted in December 2015. This paper was with the authors 1 month for 2 revisions.)
\end{abstract}

Key Words: Finite Element Method, Micro-Cantilever, Design of Experiments, Uncertainty, Robust Design

\section{INTRODUCTION}

Application of Micro-Electro-Mechanical System (aka MEMS) is expanding rapidly in different industries. Their small size that allows using them as miniature sensors and/or actuators for various purposes along with recent advancements in their large scale manufacturing provides an opportunity to easily implement them in different applications. On the other hand, there are restrictions on their mechanical design due to small size which introduces substantial differences between micro-scale and macro-scale devices. For example, using conventional actuators and joints in micro-scale is not practically possible and alternative methodologies must be considered. Electrostatic, (electro) magnetic, piezoelectric, and thermal are among the most common methods for actuation and sensing in MEMS.

Among different approaches for mechanical actuation of MEMS devices thermal actuation has the advantage of producing relatively larger amount of force and displacement as well easy implementation [1,2]. Thermal actuation is based on the thermal expansion of the component after heating. Local heat generation (e.g., via electric current) increases the temperature locally which results in expansion that in turn applies a force or extension. There is wide range of materials that can be used as a thermal actuator including most of the metals used for MEMS manufacturing. However, thermal actuation requires large amount of input power compared to other techniques in order to achieve high level of displacements [2]. Large deflection at certain points along a MEMS device can be achieved via special design to magnify the displacements produced at the thermal actuator in static/slow loading [3] or using the effect of resonance in dynamic loading [4-6]. Contrary to the macro-level actuation intuition, relatively high frequencies can be achieved using thermal actuation in micro-scale. 
This is primarily a result of considerably small thermal time constant of actuators at this scale [6]. In practice achieving periodic actuations with frequencies in order of tens of $\mathrm{MHz}$ has been confirmed by researchers $[7,8]$.

MEMS devices can be implemented in variety of architectures to accomplish different tasks for various applications [1,2]. Nonetheless, for achieving high displacement amplitudes under relatively high vibration frequencies, cantilever design can be argued as an overall optimal option to explore. It has been used in conjunction with thermal actuation effectively for various applications in the past $[4,9,10]$ and has obvious advantages in terms of simplicity in design and manufacturing. Micro-cantilevers can be excited to vibrate using several techniques such as using the bi-morph effect [5, 11], base excitation [12], electrostatic $[13,14]$ or electromagnetic [15] body forces. Using bimorph effect has been an advantageous method along with thermal actuation $[2,5]$, while the advantages of base excitation as thermal actuator has not been explored to a sufficient extent.

Goal of this research is studying the performance of a new design for the thermal actuation of a micro-cantilever used as a scanning optical fiber [16, 17]. The circular cross section and shape of the fiber (micro-cantilever beam) along with some optical requirements (e.g., no penetration or doping in the fiber) makes it hard to use seldom bimorph effect efficiently. Thus, a design relying on base excitation has been explored. The optical microcantilever is formed from extension of a thicker fiber with elevated temperature. Actuator is formed via bending a thin wire of conductive material (i.e., Aluminum). Then these two parts are attached to each other using adhesives to form the core component of the MEMS device.

This method of manufacturing brings in tolerances that might affect the overall performance of the system. Thus, the next step of this research tackles the issue of uncertainty in manufacturing and its effect on the performance of the device. This includes the effect on the natural frequency as well as dynamic response and amplitude of displacement during vibration. Finite element modeling is used for simulating the device. Modal analysis and coupled thermo-mechanical analysis is conducted in commercial finite element package of ANSYS. Then outcome are used in $2^{k}$ factorial design of experiment [18] to investigate effect of different uncertainty factors on the overall performance of the system.

\section{MICRO-CANTILEVER}

The micro-cantilever used in this study is depicted in Fig. 1. The geometrical parameters representing the critical dimensions of the system are also shown in the figure. In this figure $L$ and $d$ represent the total length and diameter of the micro-cantilever. Actuator width, height and the distance from its centre to the clamp point are shown by $w, h$ and $s$, respectively. Adhesive is applied between the micro-cantilever and the actuator. The minimum thickness of the adhesive layer is shown by $t$. As it can be seen the actuator is comprised of a bent conductive wire and is placed underneath the cantilever. Conducting electrical current through the wires results in temperature increase in the wire that results in its linear expansion. The end points of the actuator wire is connected to a base with high conductivity therefore the heat generation in the base is subtle compared to the actuator and moreover it works as a heat sink for the generated heat. Removing the current from the wire leads to cooling period in the actuator. Generally, MEMS devices have very small thermal time constants due to their extremely small sizes. This allows them to go through high frequency (up to a few $\mathrm{MHz}$ ) cycles of heating and cooling [8]. Applying repeated cycles of heating and cooling in this system will induce small displacement perturbations and vibrations in the system. Assuming that the frequency of excitation matches the fundamental frequency of the system, these perturbations result in resonance in the system. 


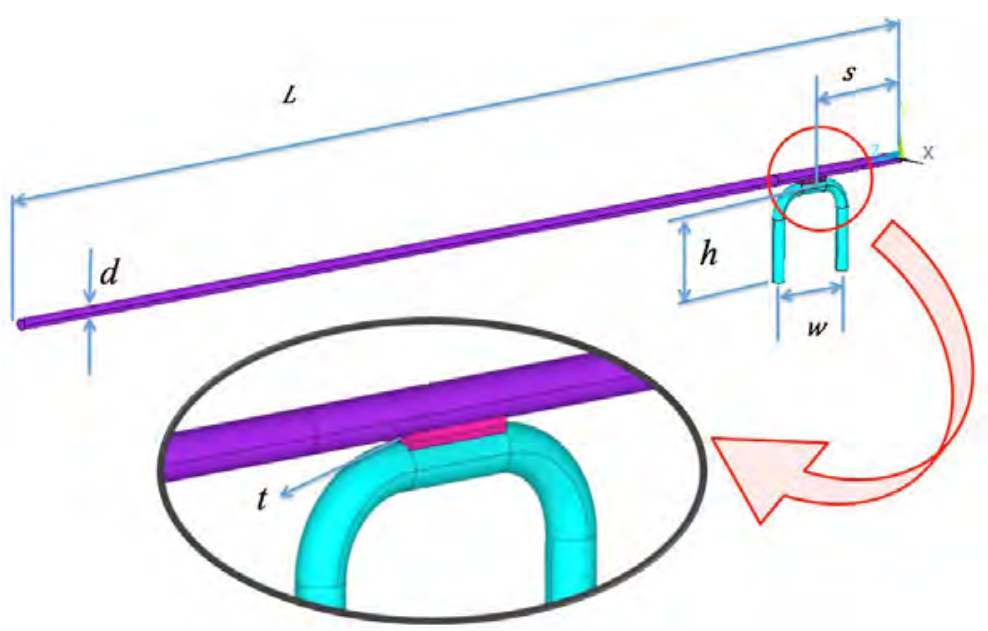

Figure 1: Geometry of the MEMS device and considered design parameters.

For practical applications, such as the case study in here, there is a certain desired frequency for the vibrations of the micro-cantilever. Therefore, the dimensions of the macrocantilever and the actuator must be set in order to match the fundamental natural frequency of the system with the required vibrational frequency. Considering there is infinite combination of parameters (length, diameter etc.) to achieve this nominal frequency it is desired to choose the values that results in a system that achieves higher deflections using lower power input.

\subsection{Finite element model}

The finite element model for the above system is constructed using ANSYS Mechanical commercial package. The meshing of the system and the boundary conditions are shown in Fig. 2. As it can be seen, in order to make the model more computationally efficient, the half model is used and corresponding boundary conditions are applied on the symmetry plane. Moreover, 3D brick elements are replaced with 3D beam elements at a certain length of the beam away from the connection points to the actuator and base. This is the region that beam is under pure bending and doesn't have considerable interaction with boundaries. Required constraints are made between the brick elements and the first beam element at the interface that this cross section change happens.
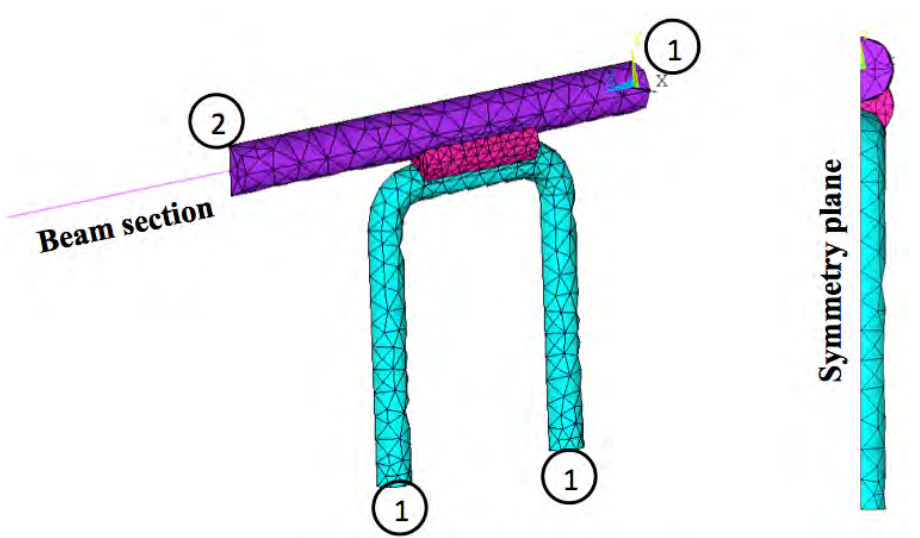
(1) Fixed surface, Temperature $=0$
(2) Rigid body constraint, connecting beam

Figure 2: The finite element model used for simulating the device (applied boundary conditions and generated mesh). 


\section{DESIGN OF EXPERIMENTS}

No matter how meticulous and detailed the design process is, the final product might have deviations from the predicted outcomes of simulations. This is an inherent characteristic of physical systems due to presence of uncertainties. In other words, the accumulated effects of tolerance and variations that are incorporated in the product during the manufacturing can results in deviations from the theoretical performance of the system. Fortunately, finite element simulation can also be used to estimate the accumulated effect of various uncertainty factors such as geometrical dimensions of the cantilever and actuator system on the overall uncertainty in the performance of the system [19]. Moreover, the effect of individual factors and the interaction between factors on the performance variations can also be determined quantitatively.

2-Level Full Factorial Design-of-Experiments is a practical approach to study the effect of uncertainties [18]. The basic idea of this methodology relies on finding (initially guessing) the higher level and lower level of the tolerances in the system. Then, a series of the experiments (design scenarios) are considered where each uncertainty factors is either at its highest value or lower value. Generating all the possible combinations of the uncertainty factors at their higher value and lower value and running all these combinations (obtaining experimental data), the effect of each factor individually as well as its interaction with other factors can be estimated [18]. Due to the fact that each factor is considered in two levels, for a total number of $k$ factors to study, there are $2^{k}$ possible combinations; hence this method is also referred to as $2^{k}$ factorial design (or 2- $k$ factorial design). This method can also be used to extract a regression function relating the effect of input parameters (geometrical factors) to the variability in the outcome (variation in the natural frequency and displacement of the microcantilever). Then, this formula allows designers to reassign/reconsider the tolerances in the input factors in order to reduce variations to a certain limit.

\subsection{Regression model}

A regression model is efficient in estimating the effect of parameters quantitatively. Moreover, it can be used to estimate the effect of changes in the system (altering the tolerances in this case) on the variability. Overall, it is an important tool in optimizing the results obtained through a full factorial design [18]. The following formula is suggested here for developing the regression model.

$$
\begin{gathered}
Y_{k}=\alpha_{0}+\sum_{i=1}^{k} \beta_{i} A_{i, m}+\sum_{i=1}^{k} \sum_{j=i+1}^{k} \gamma_{i j} A_{i, m} A_{j, m} \\
y_{m}=Y_{m}+\epsilon_{m}
\end{gathered}
$$

where $\alpha_{0}, \beta_{i}$ and $\gamma_{i j}$ are constants obtained from the regression process with $k$ being the total number of variables. $Y$ is the predicted response of the system, as opposed to $y$ which is the recorded values of system response obtained from experiments. Thus, resulting in prediction error of $\epsilon . A_{i, m}$ are the levels of input $i^{\text {th }}$ parameters (i.e., high or low) at $m^{\text {th }}$ experiment and $n=2^{k}$ is the total number of experimental points. It is preferred to normalize the input values over their range to have more coherent answers in terms of units and values. For instance here normalization is applied so that $A_{i, k}=\{-1,+1\}$. The constants in Eq. (1) can be found as described in the following:

$$
\alpha_{0}=\frac{1}{n} \sum_{k=1}^{n} y_{k}
$$




$$
\begin{gathered}
\beta_{i}=\frac{1}{n} \sum_{k=1}^{n} \Psi(i, k) y_{k} \\
\Psi(i, k)=\left\{\begin{array}{cc}
1 & \text { if } A_{i, k}=+1 \\
-1 & \text { if } A_{i, k}=-1
\end{array}\right. \\
\gamma_{i j}=\frac{1}{n} \sum_{k=1}^{n} \Phi(i, j, k) y_{k} \\
\Phi(i, j, k)=\left\{\begin{array}{cc}
\Psi(i, k) & \text { if } A_{j, k}=+1 \\
-\Psi(i, k) & \text { if } A_{j, k}=-1
\end{array}\right.
\end{gathered}
$$

\section{NUMERICAL RESULTS}

The case study in this research looks into the robust design of a micro-cantilever that is required to vibrate in a nominal frequency (i.e., $8 \mathrm{kHz}$ ). There are two criterions here that define performance: (1) the natural frequency; (2) the steady-state tip displacement during vibration. The former as mentioned above needs to be at a certain value while there is a thehigher-the-better requirement for the latter.

\subsection{Modal analysis}

The main dimensions of the micro-cantilever are set using a simple modal analysis with a few iterations. The tolerances on each geometrical factor are decided by issues such as the capabilities of manufacturing facility, acceptable costs, the resolution of the devices, etc. Table I shows the selected dimensions for achieving the required frequency and the initially suggested tolerances on each factor. It should be noted that there are unlimited combinations of dimensions that can satisfy the requirement for the frequency. Nonetheless, the variables are selected keeping in mind the importance for low flexural rigidity of the micro-cantilever as well as high length of actuator for larger deflections (as well as the typical purpose of this device). Subsequently, a dynamics simulation (as done in the next section) can provide insight on the performance of the beam in terms of its deflection.

Table I: The selected variables for achieving the nominal natural frequency and their corresponding tolerances.

\begin{tabular}{|l|c|c|c|c|c|c|}
\hline & $\begin{array}{c}\text { Fiber } \\
\text { length }(L)\end{array}$ & $\begin{array}{c}\text { Fiber diameter } \\
(d)\end{array}$ & $\begin{array}{c}\text { Actuator } \\
\text { width }(w)\end{array}$ & $\begin{array}{c}\text { Actuator } \\
\text { height }(h)\end{array}$ & $\begin{array}{c}\text { Adhesive } \\
\text { thickness }(t)\end{array}$ & $\begin{array}{c}\text { Offset } \\
(s)\end{array}$ \\
\hline Value $(\mu \mathrm{m})$ & 1180 & 14 & 70 & 80 & 1 & 20 \\
\hline Tolerances $(\mu \mathrm{m})$ & \pm 5 & \pm 0.5 & \pm 3 & \pm 3 & \pm 0.5 & \pm 2 \\
\hline
\end{tabular}

Full factorial design of experiment as it was explained above is used to investigate effect of individual factors and their interactions on natural frequency and micro-cantilever tip deflection. Finding the fundamental natural frequency for each experiment (combination of parameters), the percentage of deviation in the natural frequency from that of the nominal design (Table I) is used to evaluate the resonance frequency variations in the system.

Fig. 3 shows the direct effect of each factor on changes in the natural frequency. Plot for each parameter is generated by averaging all the values of output (frequency variations) where the studied parameter is at its normalized (over -1 to +1 range) lower and higher value (high and low levels), separately. The maximum and minimum of the frequency variations in each level is also shown using error bars. Thus, the change in the average from low level to high level is due to that particular parameter directly. Generally, factors that show a higher slope 
traveling from one level to another are more dominant factors compared to ones that have a relatively flat line.

In order to put this in a quantitative form, the regression equation formulated in Eq. (1) is used to estimate the response of the system. This results in the following function:

$$
\begin{aligned}
Y=\alpha_{0}+\beta_{L} A_{L} & +\beta_{d} A_{d}+\beta_{w} A_{w}+\beta_{h} A_{h}+\beta_{t} A_{t}+\beta_{s} A_{s}+\gamma_{L d} A_{A} A_{L} \\
& +\gamma_{L w} A_{A} A_{w}+\gamma_{L h} A_{A} A_{h}+\gamma_{L t} A_{A} A_{t}+\gamma_{L s} A_{A} A_{s}+\gamma_{d w} A_{d} A_{w} \\
& +\gamma_{d h} A_{d} A_{h}+\gamma_{d t} A_{d} A_{t}+\gamma_{d s} A_{d} A_{s}+\gamma_{w h} A_{w} A_{h}+\gamma_{w t} A_{w} A_{t} \\
& +\gamma_{w s} A_{w} A_{s}+\gamma_{h t} A_{h} A_{t}+\gamma_{h s} A_{h} A_{s}+\gamma_{t s} A_{t} A_{s}
\end{aligned}
$$
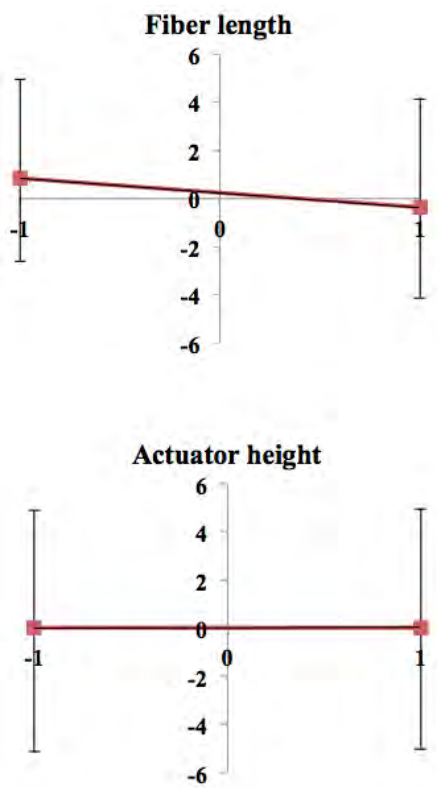
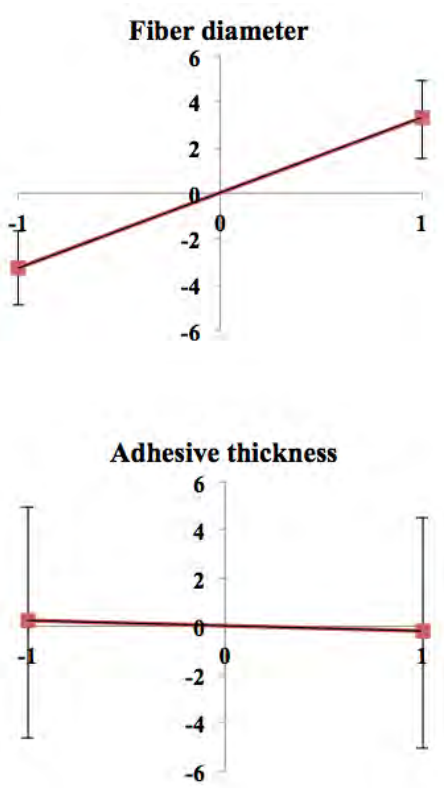
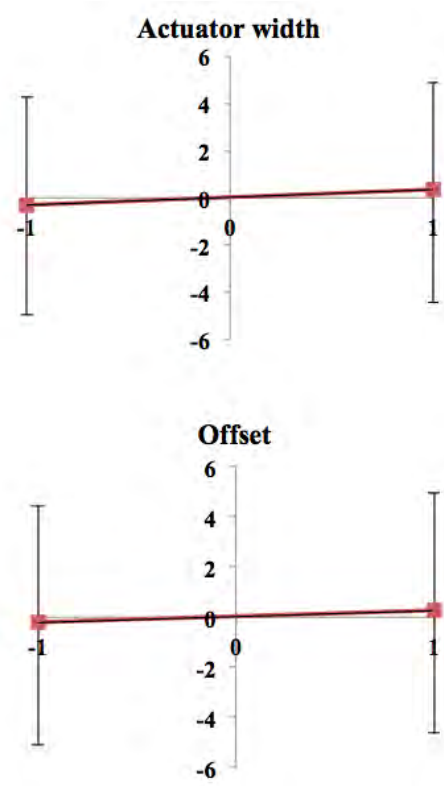

Figure 3: The direct effect of each uncertainty factor on the deviations in fundamental frequency.

Note that in this function $A_{i}$, where $i=\{L, d, w, h, t, s\}$, is the variation of the uncertainty factor (tolerance) that varies between -1 (the lower limit, normalized) and +1 (the lower limit, normalized). Using Eqs. (4) and (5), the constants for this function can be found as reported in Table II.

Table II: The direct effect of the main uncertainty parameters and their interactions on the natural frequency.

\begin{tabular}{|c|c|c|c|c|c|c|}
\hline$\alpha=5.13 \mathrm{e}-2$ & $L$ & $d$ & $w$ & $h$ & $t$ & $s$ \\
\hline$L$ & $\beta_{L}=-0.850$ & $\gamma_{L d}=-2.70 \mathrm{e}-2$ & $\gamma_{L w}=4.49 \mathrm{e}-3$ & $\gamma_{L h}=-2.34 \mathrm{e}-4$ & $\gamma_{L t}=2.66 \mathrm{e}-3$ & $\gamma_{L s}=-2.54 \mathrm{e}-3$ \\
\hline$d$ & & $\beta_{d}=3.33$ & $\gamma_{d w}=9.73 \mathrm{e}-3$ & $\gamma_{d h}=2.35 \mathrm{e}-4$ & $\gamma_{d t}=-1.95 \mathrm{e}-2$ & $\gamma_{d s}=-1.06 \mathrm{e}-3$ \\
\hline$w$ & & & $\beta_{w}=0.355$ & $\gamma_{w h}=1.80 \mathrm{e}-3$ & $\gamma_{w t}=-8.52 \mathrm{e}-3$ & $\gamma_{w s}=1.04 \mathrm{e}-2$ \\
\hline$h$ & & & & $\beta_{h}=-7.97 \mathrm{e}-3$ & $\gamma_{h t}=-5.86 \mathrm{e}-4$ & $\gamma_{h s}=-9.38 \mathrm{e}-4$ \\
\hline$t$ & & & & & $\beta_{t}=-0.246$ & $\gamma_{t s}=3.60 \mathrm{e}-3$ \\
\hline$s$ & & & & & & $\beta_{s}=0.212$ \\
\hline
\end{tabular}

Apparently, higher value (positive or negative) for a constant indicates the importance of its related direct or interaction factor, as mentioned before. Fig. 4 shows a comparison between direct effects $\left(\beta_{i}\right)$. As it can be seen in this graph as well as the values in Table II, beam diameter $(d)$ is the most important factor in controlling the natural frequency of this system in the considered range of tolerances. Therefore, in order to control the variations on the natural frequency of the final product more intense measures must be placed controlling 
the value of beam diameter. This can include using methods with higher resolution to keep the beam diameter close enough to the target value, or applying Quality Assurance (QA) methods to check each individual fiber before assembly to make sure they fall in a satisfactory range. Similar arguments can be stated for other variables that are of second and third importance in this figure and so on. Keep in mind that although elimination of uncertainties is practically impossible, it is easier to keep it contained via controlling its most dominant factors. According to Table II, the effects of the interactions $\left(\gamma_{i j}\right)$ are smaller compared to the direct effects $\left(\beta_{i}\right)$. The highest value of interaction effects according to Table II is $\gamma_{w s}=1.04 \mathrm{e}-2$. Similar to Fig. 3 the effect of interactions can be also demonstrated graphically. Fig. 5 shows the graphical representation of the interaction between offset $(s)$ and actuator width $(w)$. Here the change in the average of deviations between low and high levels of $w$ is shown while $s$ is kept at its low/high level. The change in the slope between these two lines indicates the effect of interaction [18]. As it can be seen in this figure, there is a negligible change in the slope of line when changing $w$ at different levels of $s$ (less than $2 \%$ ). Therefore, it can be argued that the effect of interactions is insignificant.

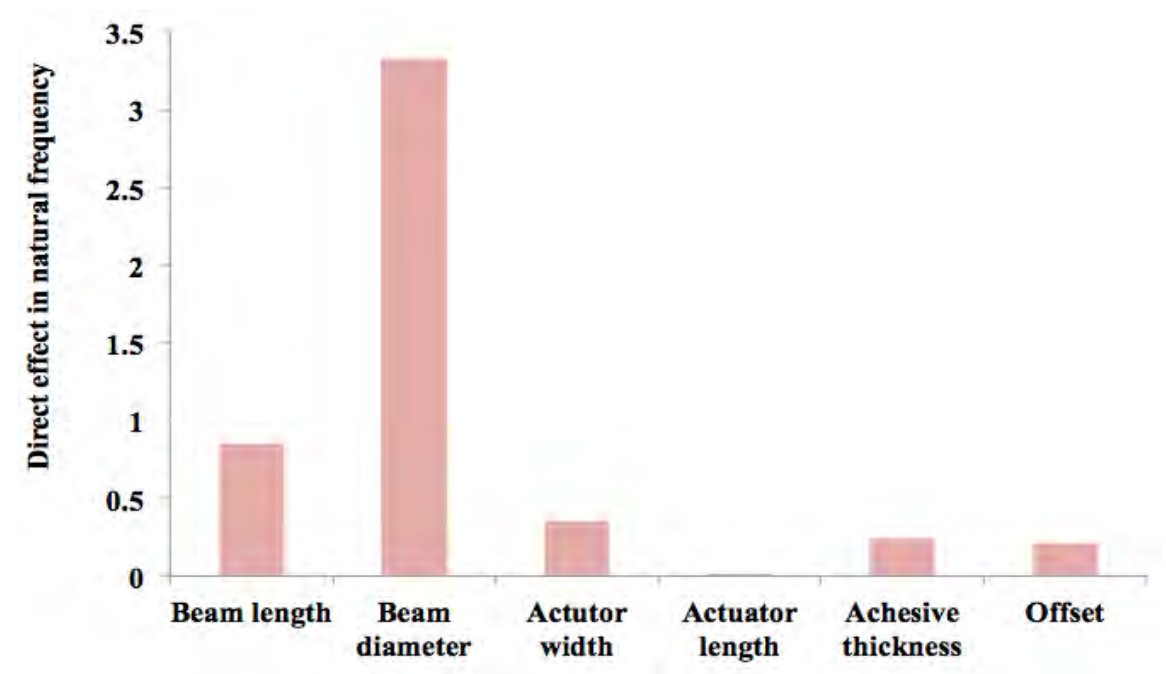

Figure 4: Direct effect of uncertainty factors on fundamental frequency.

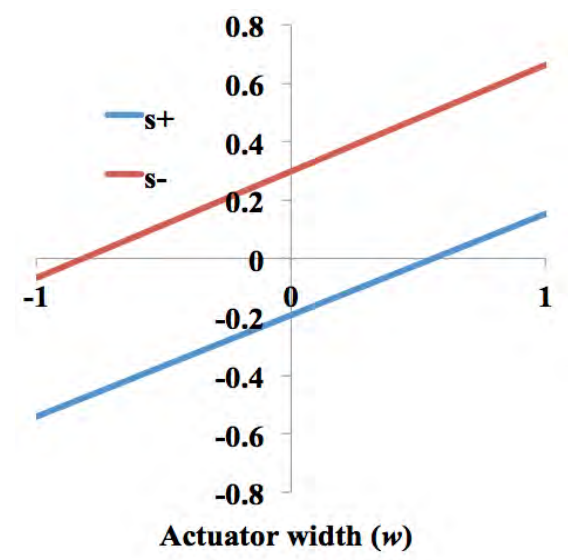

Figure 5: Interaction between actuator width and offset in variations in fundamental frequency.

\subsection{Coupled thermo-mechanical analysis}

The tip displacement of the micro-cantilever and the effect of uncertainty and tolerances on its variation is studied next. As discussed previously, thermal cycles and the effect of expansions/contractions in the actuator is the main source of actuation. Heating of the actuator can be achieved for instance by conducting electric current through wires. This results in 
internal body heat generation in the actuator wires. Because both positive and negative magnitudes of an alternating current induce heat in the wire, only one half of the AC current should be applied and the other half must be eliminated. Absence of current in the second half of cycle allows the wire/actuator to cool down and completes the heating/cooling cycles. Accordingly, the following formula is implemented in the finite element simulation:

$$
b(\tau)=q \frac{\sin (2 \pi f \tau)+|\sin (2 \pi f \tau)|}{2}
$$

where $q$ is the constant amplitude of heat generation, $f$ frequency of excitation, and $\tau$ represents time. The effective amount of power used in the actuator $\left(P_{\text {ave }}\right)$ can be calculated via:

$$
P_{\text {ave }}=\frac{1}{T} \int_{0}^{T} q \frac{\sin (2 \pi f \tau)+|\sin (2 \pi f \tau)|}{2} V d \tau=\frac{q V}{\pi}
$$

where $T=f^{-1}$ is the period of vibration and $V$ is the volume of actuator.

The transient (time-history) thermo-mechanical response of the system to above internal heat generation function is achieved using ANSYS/Mechanical finite element package. The analysis is continued for long enough to obtain steady state response in the system in terms of tip displacement and temperature changes in the variable. Preliminary simulations show that this condition is attained at $\sim 500$ cycles. Fig. 6 shows tip displacement in the nominal design. As it can be seen this cantilever can obtain a tip displacement amplitude of $\sim 82$ micron at 8 $\mathrm{kHz}$ with $10 \mathrm{~mW}$ input power.

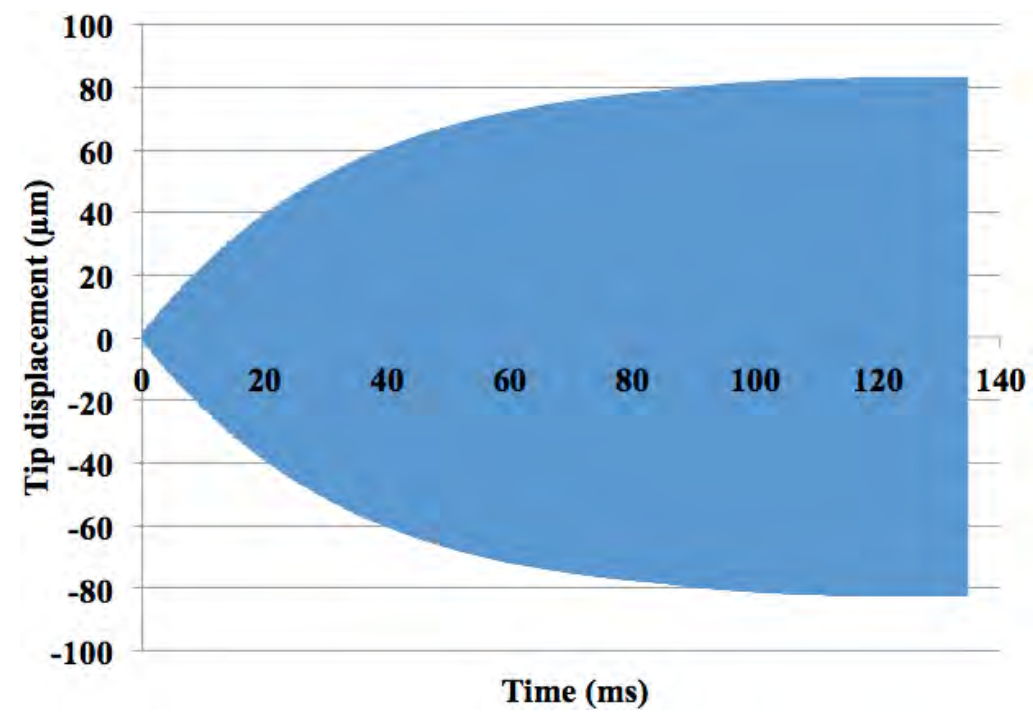

Figure 6: Tip displacement for the nominal design with $10 \mathrm{~mW}$ input power.

Similar arguments to section 4.1 can be made for the effect of tolerances and uncertainty factors on the variation of this tip displacement value. The difference here compared to the variations in the natural frequency is that, in that case variation from the target frequency $(8$ $\mathrm{kHz}$ ) was not desirable regardless of it being higher or lower than the target (positive or negative output in the regression function). However, in the case of tip displacement only reduction in the tip displacement amplitude is undesirable and if the tolerances in the manufacturing lead to increase in the tip displacement they are acceptable, as they improve the overall performance.

Fig. 7 illustrates the change in the average at high and low level of each individual factor, as well as the maximum and minimum bounds of variation at each level. The general form of Eq. (6) is used again to find the direct effects $\left(\beta_{i}\right)$ and interactions $\left(\gamma_{i j}\right)$, and results are shown 
in Table III. Fig. 8 shows a comparison between magnitudes of different factors in the variation of displacement magnitudes.
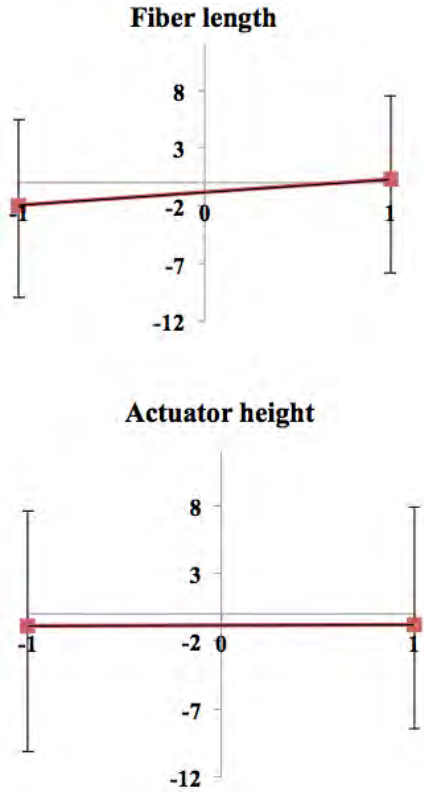

Fiber diameter

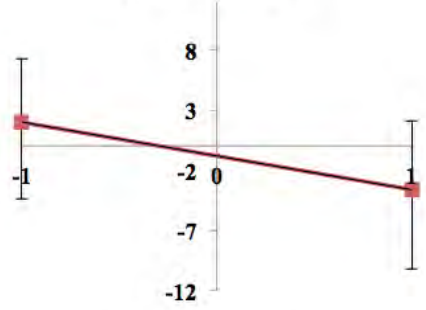

Adhesive thickness

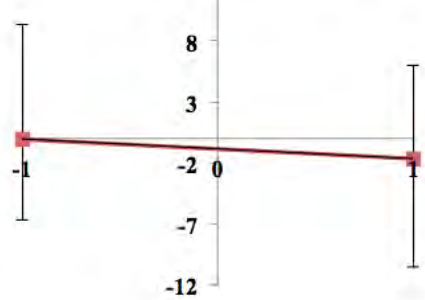

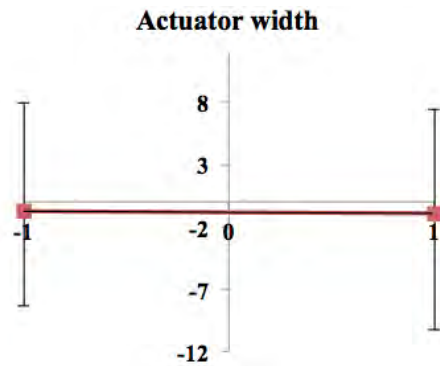

Offset

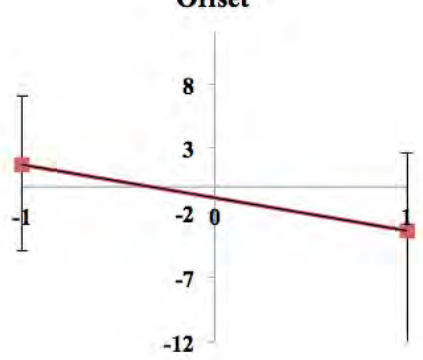

Figure 7: The direct effect of each uncertainty factor on the deviations in steady-state tip displacement.

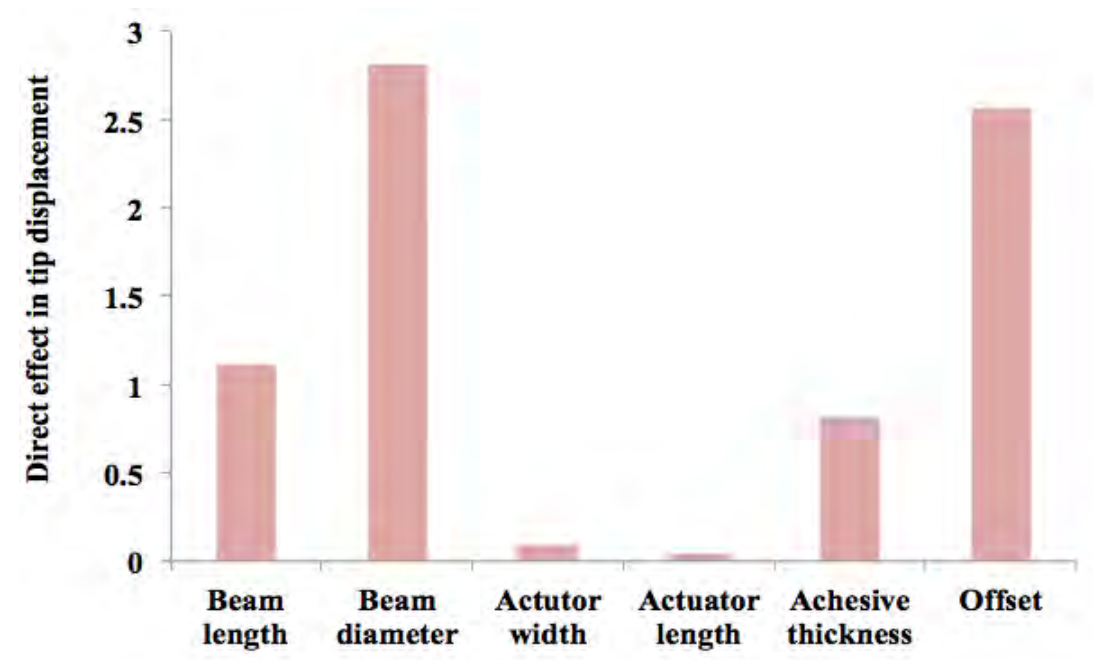

Figure 8: Direct effect of uncertainty factors on steady-state tip displacement.

Table III: The direct effect of the main uncertainty parameters and their interactions on the tip displacement.

\begin{tabular}{|c|c|c|c|c|c|c|}
\hline$\alpha=-0.840$ & $L$ & $d$ & $w$ & $h$ & $t$ & $s$ \\
\hline$L$ & $\beta_{L}=1.11$ & $\gamma_{L D}=-2.91 \mathrm{e}-2$ & $\gamma_{L w}=3.78 \mathrm{e}-2$ & $\gamma_{L h}=-4.59 \mathrm{e}-2$ & $\gamma_{L t}=7.59 \mathrm{e}-3$ & $\gamma_{L s}=6.89 \mathrm{e}-2$ \\
\hline$d$ & & $\beta_{d}=-2.81$ & $\gamma_{d w}=-0.29$ & $\gamma_{d h}=0.101$ & $\gamma_{d t}=0.74$ & $\gamma_{d s}=-0.457$ \\
\hline$w$ & & & $\beta_{w}=-9.04 \mathrm{e}-2$ & $\gamma_{w h}=-0.138$ & $\gamma_{w t}=-0.906$ & $\gamma_{w h}=0.757$ \\
\hline$h$ & & & & $\beta_{h}=4.48 \mathrm{e}-2$ & $\gamma_{h t}=0.269$ & $\gamma_{h s}=2.22 \mathrm{e}-4$ \\
\hline$t$ & & & & & $\beta_{t}=-0.814$ & $\gamma_{t s}=-0.228$ \\
\hline$s$ & & & & & & $\beta_{s}=-2.56$ \\
\hline
\end{tabular}

It can be argued that the direct effect of beam length and diameter are of significant contribution to the variations. Also, the adhesive thickness and offset value that were insignificant in the variation of natural frequency can have noticeable impact on the tip 
displacement. Moreover, the interaction between some factors (e.g., $\gamma_{w t}$ and $\gamma_{d w}$ ) show relatively important effect on the variations in the tip displacement. Fig. 9 shows the interaction between actuator width and adhesive thickness graphically. As it can be seen in this figure there is a substantial change $(\sim 85 \%)$ in the slope of lines. This indicates the variation in tip displacement due to actuator width $(w)$ depends on adhesive thickness $(t)$, and vice versa.

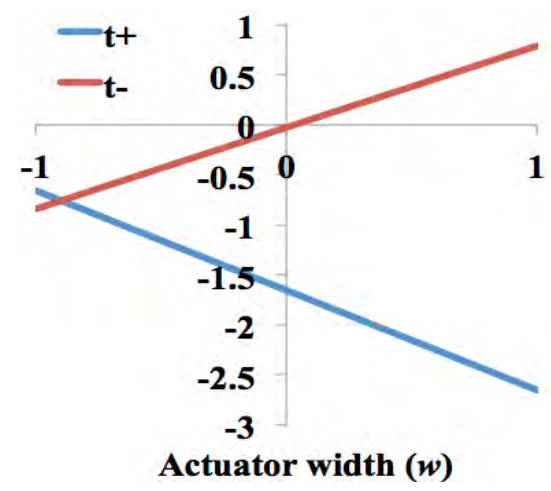

Figure 9: Interaction between actuator width and offset in variations in steady-state tip displacement.

\subsection{Analysis of variability and robust design}

Results from sections 4.1 and 4.2 can be used to draw suggestion and conclusions on the robust design of the current micro-cantilever system. The regression functions (variability models) that are generated for the deviations in the fundamental frequency and tip displacements, according to Eq. (6) is used to predict the response of system if the input factors are changed within the analysis range. Therefore, it can be argued that by limiting the tolerances of each individual variable its impact on the variability of fundamental frequency and tip displacement can be estimated using Eq. (6) along with constants in Tables II and III.

For instance, it is known from Figs. 4 and 8 that beam diameter (in its current tolerance range) is the most significant factor in affecting the variations in both fundamental frequency and tip displacement. Generally, if beam diameter is higher/lower than the nominal value the natural frequency increases/decreases while displacement decreases/increases. On the hand, offset value shows small influence on the natural frequency while it has a considerable role in tip displacement variation. Here, increase in the offset value results in decrease in tip displacement. Based on these a simple strategy to improve the robustness of this product is put forward as a possible scenario.

In order to keep the natural frequency on the target, the methods for manufacturing the beam can be modified to higher resolution methods to improve the manufactured beam diameter accuracy. In addition, quality assurance can be applied to discard beams with diameters falling out of the designated tolerance. On the other hand, the tip displacement is largely affected by the offset value and as it is evident in Fig. 7, an increase in the offset value decreases the tip displacement. In this paper only these two factors are studied for reducing the variability (increasing robustness); nonetheless, similar arguments can be made for more factors.

Because of the fact that beam diameter changes the fundamental frequency in general and it is required to satisfy vibration in a certain frequency, it is desirable to decrease the tolerances of the beam diameter. However, offset value only has an adverse effect when it is on its positive tolerance values. Let's assume in the modified design the tolerances on the beam diameter in the final manufactured system is improved by $60 \%$ (bringing it to $\pm 0.2 \mu \mathrm{m}$ instead of $\pm 0.5 \mu \mathrm{m}$ ) and only accepting the negative tolerance values on the offset parameter ( $-2 \mu \mathrm{m}$ instead of $\pm 2 \mu \mathrm{m})$. Without any further need to run the simulations, the regression 
models used in previous sections can be used to estimate variations in the fundamental frequency as well as tip displacement after implementing the new tolerance rules.

Fig. 10 shows all the scattered virtual experiment (simulation) data points calculated using the finite element method (blue diamonds) in a plane of deviation in fundamental frequency (horizontal axis) and tip displacement (vertical axis). As it can be seen the fundamental frequency and tip displacements show a variation of around $\pm 5 \%$ and $\pm 9 \%$, respectively.

The effect of modification in these variations is shown by applying the new tolerance mentioned above. Re-generating all the design points at the high level and low levels of their new tolerance range and estimating the response using the regression function results in red squares shown in Fig. 10. As it can be seen, the overall scattering of the results is reduced to around $\pm 3 \%$ and $\pm 5 \%$, for fundamental frequency and tip displacement respectively. Moreover, the distribution of points at the plane shows that for the tip displacement, $\sim 10 \%$ of points are moved from the negative side of the tip displacement variation (lower than nominal design) to the positive side (higher than nominal design). This effect which is a desirable phenomenon can be attributed to the asymmetric tolerances on the offset value which only eliminated the tolerance values that were adversely affecting the variations.

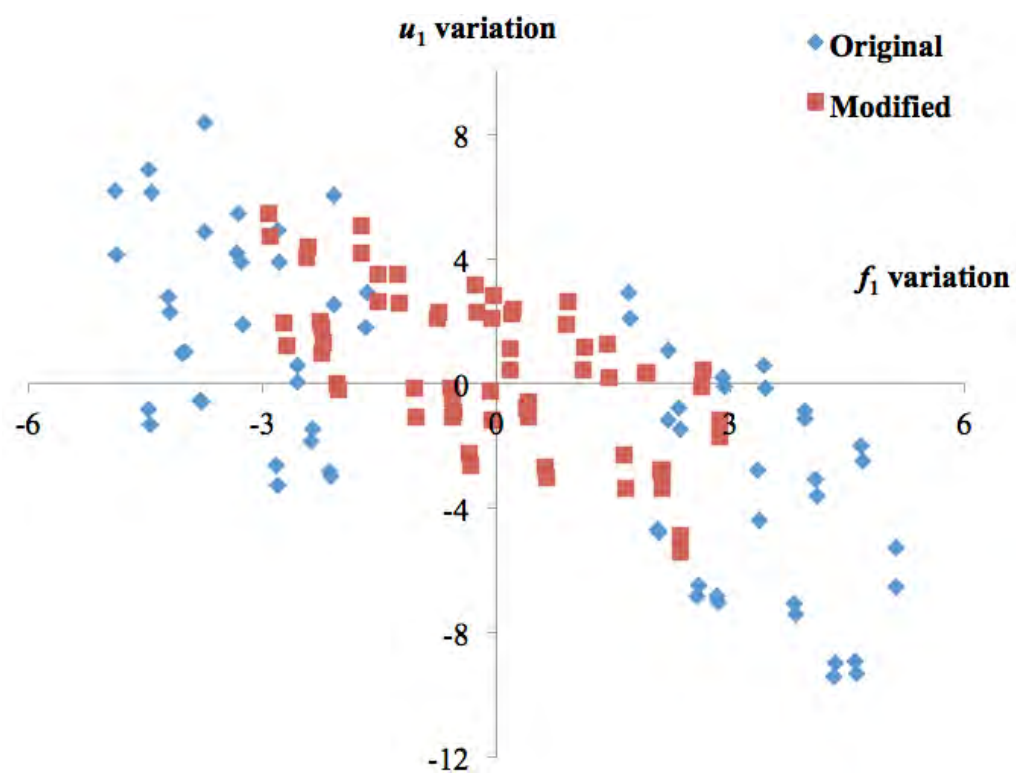

Figure 10: Virtual experiment points variability before and after modifying the tolerances.

\section{CONCLUSIONS AND REMARKS}

The robust design of a vibrating micro-cantilever using thermal actuation was presented here. The actuation relies on the thermal expansion/contraction of an actuator located close to the clamp point of the micro-cantilever. Small perturbations at this point that matches the fundamental frequency of the system can result in large deflections due to effect of resonance.

The performance requirements for a given purpose needs this device to operate in a particular nominal frequency and deviation from that frequency result in a deterioration of overall performance. Moreover, the higher tip displacement amplitude for a certain input power is a certain benefit in terms of efficiency while the opposite is not desirable. Therefore, a full factorial design of experiment methodology is pursued here in order to decrease variability and improve the efficiency of the system via controlling geometrical tolerances.

The first design and analysis on system was carried out to reach at a nominal design. However, it is evident that landing on the exact value of geometrical factors is practically impossible. An initial set of tolerances is used to investigate the overall robustness of the 
system. Two-level $\left(2^{k}\right)$ full factorial design of experiment is implemented to achieve this goal. Separate analysis on the deviation of fundamental frequency and tip displacements under the effect of tolerances is conducted to estimate the importance of each geometrical factor on the variation from the nominal design. ANSYS finite element software is used to calculate the natural frequency and the transient (time-dependent) thermo-mechanical response of the system. A general regression function based on the extracted data points is generated to represent the response of system in further design iterations. Results show that for the fundamental frequency, the assumed tolerances for beam diameter and length are the factors that can change the outcome by around $1 \%$ or more, while the latter parameters along with adhesive thickness and the distance of actuator from the clamp point (offset) are factors that have close to $1 \%$ or more deviation in the tip displacement. Although, $1 \%$ might seem negligible for one factor, note that the combined effect of all factors together can induce up to $\sim 9 \%$ reduction in performance of the system. Moreover, where the interaction between various factors doesn't show significant contribution in the variations of natural frequency (less than $0.01 \%$ ), it has more effect between some factors in the variation of tip displacement (e.g., $0.9 \%$ due to combination of actuator width and adhesive thickness).

The extracted regression functions are used to predict the variations on the fundamental frequency and tip displacement after modifying the tolerances. This is used to modify the design tolerances in order to reduce the variability in the system. Note that, the goal is to reduce the variability in the fundamental frequency (symmetric) and avoid the reduction in the tip displacement due to tolerances (asymmetric). For here, modifications on tolerances on beam diameter is selected as its value affects the fundamental frequency and tip displacement significantly; and the tolerance in offset as a parameter that affects tip displacement notably while having negligible effect on the fundamental frequency. The tolerance in the former is reduced symmetrically and for the latter only tolerance on the side that result in increasing the tip displacement is passed as acceptable. Estimating the variation in the performance of system using the regression functions and new tolerances shows $\sim 40 \%$ reduction in the variations of fundamental frequency and $\sim 55 \%$ reduction in the drop in tip displacements due to effect of tolerances.

To summarize, this procedure for robust design can be depicted as a flowchart shown in Fig. 11.

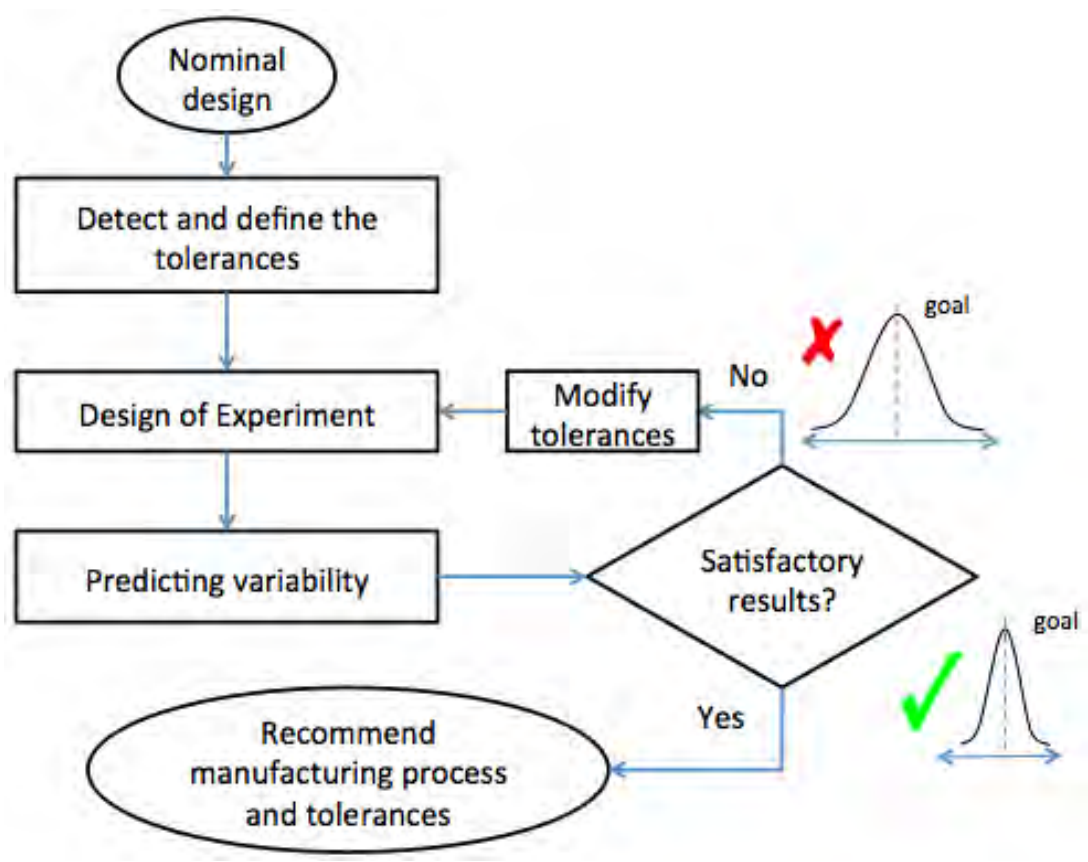

Figure 11: Flowchart of the robust design process used in this research. 
The process starts with a nominal design that satisfies the basic requirements. Then, the sources of uncertainty in the system (e.g., tolerances) are detected and their initial values based on the capabilities of the manufacturing process, cost of operations, quality assurance etc. are determined. Then the full design of experiment is conducted to predict the overall variations in the system response (e.g., resonance frequency and tip displacement in here). Results are processed to determine the importance of each individual factor as well as interactions between them. Afterwards, decisions can be made on controlling factors that are contributing to the overall variation significantly. Controlling these factors can reduce the variations in the system to a large extent. Thus, new measures are applied to control them and reduce the variability. This can be done symmetrically (theoretical goal is reducing it to zero), or asymmetrically (only positive or negative side of the tolerance is reduced/eliminated). Here the regression function is used to estimate the response of system using new tolerances in a new iteration of full factorial design. Depending on whether the satisfactory results in terms of system robustness is attained or not, this process can be continued.

\section{ACKNOWLEDGEMENT}

The Natural Sciences and Engineering Research Council of Canada (NSERC), the Canadian Institutes of Health Research (CHIR) and the Michael Smith Foundation supported this study for Health Research (MSFHR). Authors would also like to acknowledge the Python Software Foundation and particularly the providers of Anaconda Scientific Python distribution, as well as NumPy and SciPy libraries. The majority of numerical work in this paper was run using the open source packages provided by these communities. We would also like to acknowledge CMC Microsystems for the providing ANSYS academic package that facilitated this research.

\section{REFERENCES}

[1] Maluf, N.; Williams, K. (2004). An introduction to microelectromechanical systems engineering, $2^{\text {nd }}$ ed., Artech House, Inc., Boston

[2] Lobontiu, N.; Garcia, E. (2005). Mechanics of microelectromechanical systems, Kluwer Academic Publishers, Boston

[3] Kolesar, E. S.; Allen, P. B.; Howard, J. T.; Wilken, J. M.; Boydston, N. (1999). Thermallyactuated cantilever beam for achieving large in-plane mechanical deflections, Thin Solid Films, Vol. 356, 295-302

[4] Schweizer, S.; Cousseau, P.; Lammel, G.; Calmes, S.; Renaud, P. (2000). Two-dimensional thermally actuated optical microprojector, Sensors and Actuators A: Physical, Vol. 85, No. 1-3, 424-429, doi:10.1016/S0924-4247(00)00384-8

[5] Zhang, Y.-W.; Zhang, Y.-X.; Marcus, R. B. (1999). Thermally actuated microprobes for a new wafer probe card, Journal of Microelectromechanical Systems, Vol. 8, No. 1, 43-49, doi: $10.1109 / 84.749401$

[6] Sehr, H.; Tomlin, I. S.; Huang, B.; Beeby, S. P.; Evans, A. G. R.; Brunnschweiler, A.; Ensell, G. J.; Schabmueller, C. G. J.; Niblock, T. E. G. (2002). Time constant and lateral resonances of thermal vertical bimorph actuators, Journal of Micromechanics and Microengineering, Vol. 12, No. 4, 410-413, doi:10.1088/0960-1317/12/4/311

[7] Rahafrooz, A.; Hajjam, A.; Tousifar, B.; Pourkamali, S. (2010). Thermal actuation, a suitable mechanism for high frequency electromechanical resonators, IEEE $23^{\text {rd }}$ International Conference on Micro Electro Mechanical Systems (MEMS), 200-203, doi:10.1109/MEMSYS.2010.5442530

[8] Rahafrooz, A.; Pourkamali, S. (2011). High-frequency thermally actuated electromechanical resonators with piezoresistive readout, IEEE Transactions on Electron Devices, Vol. 58, No. 4, 1205-1214, doi:10.1109/TED.2011.2105491

[9] Grigorov, A.; Davis, Z. J.; Rasmussen, P.; Boisen, A. (2004). A longitudinal thermal actuation principle for mass detection using a resonant micro-cantilever in a fluid medium, Microelectronics Engineering, Vol. 73-74, 881-886, doi:10.1016/j.mee.2004.03.069 
[10] Schweizer, S.; Calmes, S.; Laudon, M.; Renaud, P. (1999). Thermally actuated optical microscanner with large angle and low consumption, Sensors and Actuators A: Physical, Vol. 76, No. 1-3, 470-477, doi:10.1016/S0924-4247(99)00012-6

[11] Ataka, M.; Omodaka, A.; Takeshima, N.; Fujita, H. (1993). Fabrication and operation of polyimide bimorph actuators for a ciliary motion system, Journal of Microelectromechanical Systems, Vol. 2, No. 4, 146-150, doi:10.1109/84.273089

[12] Zhang, W.; Meng, G.; Li, H. (2006). Adaptive vibration control of micro-cantilever beam with piezoelectric actuator in MEMS, International Journal of Advanced Manufacturing Technology, Vol. 28, No. 3, 321-327, doi:10.1007/s00170-004-2363-5

[13] Dong, J.; Ferreira, P. M. (2009). Electrostatically actuated cantilever with SOI-MEMS parallel kinematic XY stage, Journal of Microelectromechanical Systems, Vol. 18, No. 3, 641-651, doi:10.1109/JMEMS.2009.2020371

[14] Liu, S.; Davidson, A.; Lin, Q. (2004). Simulation studies on nonlinear dynamics and chaos in a MEMS cantilever control system, Journal of Micromechanics and Microengineering, Vol. 14, No. 7, 1064-1073, doi:10.1088/0960-1317/14/7/029

[15] Lange, D.; Hagleitner, C.; Herzog, C.; Brand, O.; Baltes, H. (2003). Electromagnetic actuation and MOS-transistor sensing for CMOS-integrated micromechanical resonators, Sensors and Actuators A: Physical, Vol. 103, No. 1-2, 150-155, doi:10.1016/S0924-4247(02)00307-2

[16] Seibel, E. J.; Smithwick, Q. Y. J. (2002). Unique features of optical scanning, single fiber endoscopy, Lasers in Surgery and Medicine, Vol. 30, No. 3, 177-183, doi:10.1002/1sm.10029

[17] Lee, C. M.; Engelbrecht, C. J.; Soper, T. D.; Helmchen, F.; Seibel, E. J. (2010). Scanning fiber endoscopy with highly flexible, $1 \mathrm{~mm}$ catheterscopes for wide-field, full-color imaging, Journal of Biophotonics, Vol. 3, No. 5-6, 385-407, doi:10.1002/jbio.200900087

[18] Montgomery, D. C. (2012). Design and Analysis of Experiments, Wiley, Boston

[19] Komeili, M.; Milani, A. S. (2012). The effect of meso-level uncertainties on the mechanical response of woven fabric composites under axial loading, Computers \& Structures, Vol. 90-91, 163-171, doi:10.1016/j.compstruc.2011.09.001 